\title{
Renormalization of two-loop diagrams in scalar lattice field theory
}

\author{
B. Borasoy ${ }^{1}$ and H. Krebs ${ }^{2}$ \\ Helmholtz-Institut für Strahlen- und Kernphysik (Theorie), \\ Universität Bonn, Nußallee 14-16, D-53115 Bonn, Germany
}

\begin{abstract}
We present a method to calculate to very high precision the coefficients of the divergences occuring in two-loop diagrams for a massive scalar field on the lattice. The approach is based on coordinate space techniques and extensive use of the precisely known Green's function.
\end{abstract}

PACS: $12.90 .+b$

\footnotetext{
${ }^{1}$ email: borasoy@itkp.uni-bonn.de

${ }^{2}$ email: hkrebs@itkp.uni-bonn.de
} 


\section{Introduction}

Lattice regularization is a convenient tool to study both the perturbative and non-perturbative aspects of the underlying theory. Within this framework the action is formulated on a discretized space-time lattice and the partition function can be evaluated numerically, e.g., by employing Monte Carlo simulations. This feature of lattice regularization allows one to study the non-perturbative regime of a theory and is thus of particular interest for QCD at low energies.

Eventually, however, one is interested in performing the continuum limit by letting the lattice spacing approach zero. In order to establish the correct link between numerical lattice simulations and the physical continuum, perturbative lattice calculations prove useful. They are, e.g., necessary to calculate the renormalization of the couplings in the Lagrangian or the renormalization factors of operator matrix elements. In general, it allows one to study perturbatively how simulation results approach continuum results. The discretization effects, i.e., the corrections due to the finite lattice spacing, can also be investigated and this knowledge may help to reduce the pertinent systematic error in lattice results.

The explicit calculation of loop diagrams in lattice regularization turns out to be more tedious than in conventional continuum regularization schemes such as dimensional regularization. In this respect, coordinate space methods have proven useful in the evaluation of Feynman diagrams and allow a very precise determination of the continuum limit of one- and two-loop integrals [1]. In [1] this technique was applied to massless propagators. In [2] the formalism of [1] was extended to massive scalar fields by presenting an efficient method to calculate the associated Green's function to very high precision.

In the present investigation, we will show that the precise knowledge of the Green's function is helpful in extracting the divergent pieces of two-loop diagrams for massive scalar fields to very high accuracy. The method developed is therefore suited to calculate the renormalization of the bare Lagrangian parameters at the two-loop level.

This work is organized as follows. First, external momenta in the loop propagators are eliminated by applying the BPHZ scheme on the lattice so that the discussion can be reduced to sunset-type loops without external momenta in the propagators. We illustrate the BPHZ method in the next section for the basic sunset diagram. Then, the divergences of the reduced integrals are evaluated in Sec. 3 by applying coordinate space methods. In Sec. 4 we present the numerical results for the quadratic and logarithmic would-be divergences of the sunset diagram and in Sec. 5 we provide additional relations between the logarithmic pieces and oneloop tadpole diagrams. We summarize our findings in Sec. 6. Technicalities are deferred to the appendices.

\section{BPHZ scheme on the lattice}

Within the BPHZ scheme the ultraviolet divergences of integrals are removed by performing subtractions directly in the integrand of a Feynman integral [3]. This is achieved by subtracting the first few terms of the Taylor expansion in the external momenta. Originally this method was developed for the continuum formulation of field theories, but afterwards extended to lattice field theories as well [4].

In the present work we are particularly interested in isolating the divergent pieces of a 


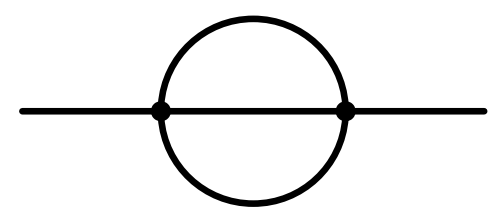

Figure 1: Shown is the sunset diagram with three massive propagators.

given lattice loop integral, while the remaining finite pieces can be calculated numerically for a given lattice spacing. We restrict ourselves to massive scalar field theories on the lattice. For illustration the method will be worked out explicitly for the basic sunset diagram which arises in $\phi^{4}$ theory at the two-loop level, see Fig. 1, but a general two-loop diagram can be evaluated in the same manner. The first step in the approach is the application of the BPHZ scheme so that the would-be divergences which occur as the lattice spacing goes to zero are contained in lattice integrals without external momenta, while the momentum-dependent but finite remainder can be neglected for renormalization purposes. Provided the lattice theory satisfies the power counting conditions given in [5], which is the case here, the continuum limit of these momentum-dependent finite pieces exists, is independent of the details of the lattice action and is identical with the BPHZ finite parts obtained from the corresponding continuum action [4]. In order to illustrate the method, we will confirm explicitly for the case of the sunset diagram that this remainder is indeed finite in the continuum limit. For definiteness we work with a hypercubical lattice $\Lambda$ of infinite volume and the standard free scalar propagator, while the results are presented in lattice units if not otherwise stated.

The basic sunset diagram for a massive scalar field with external momentum $p$ is given by Fig. 1,

$$
\int_{-\pi}^{\pi} \frac{d^{4} k}{(2 \pi)^{4}} \frac{d^{4} q}{(2 \pi)^{4}} I_{S}(k, q ; p)=\int_{-\pi}^{\pi} \frac{d^{4} k}{(2 \pi)^{4}} \frac{d^{4} q}{(2 \pi)^{4}} \frac{1}{\Delta(k) \Delta(q) \Delta(p+k+q)},
$$

where the subscript $S$ of $I$ denotes the full sunset diagram and $\Delta$ is the inverse lattice propagator

$$
\Delta(p)=m^{2}+\hat{p}^{2}=m^{2}+\sum_{\mu=1}^{4} \hat{p}_{\mu}^{2}=m^{2}+4 \sum_{\mu=1}^{4} \sin ^{2}\left(\frac{p_{\mu}}{2}\right) .
$$

Let $\gamma_{1}, \gamma_{2}, \gamma_{3}$ be the three subdiagrams of $S$, Fig. 2, then the corresponding set of forests is given by $\mathcal{F}(S)=\left\{\emptyset, S, \gamma_{1}, \gamma_{2}, \gamma_{3}, S \gamma_{1}, S \gamma_{2}, S \gamma_{3}\right\}$ with the superficial degrees of divergence $d(S)=2$ and $d(\gamma)=0$ otherwise. The integrand $I_{S}$ of the sunset diagram leads to would-be divergences in the continuum limit as can be seen by explicitly inserting the lattice spacing in Eq. (1). A convenient renormalization procedure to handle these divergences is provided by the forest formula of Zimmermann [3] wherein a subtraction operation is directly applied to

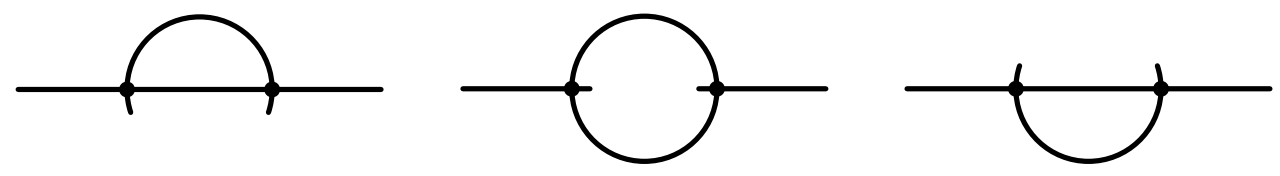

Figure 2: Subdiagrams $\gamma_{1,2,3}$ of the sunset diagram. 
the integrand. Within this approach the renormalized Feynman integrand $R_{S}$ is given by

$$
R_{S}=\sum_{\Gamma \in \mathcal{F}} \prod_{\gamma \in \Gamma}\left(-t_{\gamma}^{d(\gamma)}\right) I_{S}
$$

where $t_{\gamma}^{d(\gamma)}$ are lattice subtraction operators of order $d(\gamma)$ as introduced in [4]. In the continuum limit the operators $t_{\gamma}^{d(\gamma)}$ coincide with the first $d(\gamma)$ terms of the Taylor expansion of the integrand $I_{\gamma}$ in the external momentum of the subgraph. For the sunset diagram one obtains

$$
\begin{aligned}
R_{S} & =\left(1-t_{S}^{2}-t_{\gamma_{1}}^{0}-t_{\gamma_{2}}^{0}-t_{\gamma_{3}}^{0}+t_{S}^{2}\left[t_{\gamma_{1}}^{0}+t_{\gamma_{2}}^{0}+t_{\gamma_{3}}^{0}\right]\right) I_{S} \\
& =\left(1-t_{S}^{2}-\left[1-t_{S}^{2}\right]\left[t_{\gamma_{1}}^{0}+t_{\gamma_{2}}^{0}+t_{\gamma_{3}}^{0}\right]\right) I_{S}
\end{aligned}
$$

Since $\left[1-t_{S}^{2}\right]\left(t_{\gamma_{1}}^{0}+t_{\gamma_{2}}^{0}+t_{\gamma_{3}}^{0}\right) I_{S}=0$, this leads to

$$
R_{S}=\left[1-t_{S}^{2}\right] I_{S}
$$

A convenient choice for the subtraction operator $t_{S}^{2}$ is given by [4]

$$
t_{S}^{2} I_{S}(k, q ; p)=I_{S}(k, q ; 0)+\sum_{\mu} \sin p_{\mu}\left(\frac{\partial}{\partial p_{\mu}} I_{S}\right)_{p=0}+\frac{1}{2} \sum_{\mu, \nu} \sin p_{\mu} \sin p_{\nu}\left(\frac{\partial}{\partial p_{\mu}} \frac{\partial}{\partial p_{\nu}} I_{S}\right)_{p=0}
$$

where the partial derivatives are to be taken at $p=0$. This yields

$$
\begin{aligned}
t_{S}^{2} I_{S}(k, q ; p)=\Delta^{-1}(k) \Delta^{-1}(q) \Delta^{-1}(k+q) & \left(1-\sum_{\mu} \sin ^{2} p_{\mu} \cos (k+q)_{\mu} \Delta^{-1}(k+q)\right. \\
& \left.+4 \sum_{\mu} \sin ^{2} p_{\mu} \sin ^{2}(k+q)_{\mu} \Delta^{-2}(k+q)\right) .
\end{aligned}
$$

Utilizing hypercubical symmetry of the lattice it can be rewritten as

$$
\begin{aligned}
t_{S}^{2} I_{S}(k, q ; p)=\Delta^{-1}(k) \Delta^{-1}(q) \Delta^{-1}(k+q) & \left(1+\frac{1}{8} \bar{p}^{2}-\left(1+\frac{m^{2}}{8}\right) \bar{p}^{2} \Delta^{-1}(k+q)\right. \\
& \left.+4 \sum_{\mu} \bar{p}_{\mu}^{2}(\overline{k+q})_{\mu}^{2} \Delta^{-2}(k+q)\right)
\end{aligned}
$$

with

$$
\bar{p}^{2}=\sum_{\mu=1}^{4} \bar{p}_{\mu}^{2}=\sum_{\mu=1}^{4} \sin ^{2} p_{\mu} .
$$

Neglecting pieces that do not lead to divergencies in the continuum limit this simplifies to

$$
\begin{aligned}
t_{S}^{2} I_{S}(k, q ; p)=\Delta^{-1}(k) \Delta^{-1}(q) \Delta^{-1}(k+q) & \left(1-\bar{p}^{2} \Delta^{-1}(k+q)\right. \\
& \left.+4 \sum_{\mu} \bar{p}_{\mu}^{2}(\overline{k+q})_{\mu}^{2} \Delta^{-2}(k+q)\right)+\ldots
\end{aligned} .
$$


According to [4] integration of $R_{S}$ in Eq. (5) yields a finite continuum limit which is independent of the details of the lattice action. In order to demonstrate the finiteness of the renormalized integral, it is thus sufficient to consider the corresponding integral in the continuum formulation

$$
\begin{aligned}
& \int_{-\infty}^{\infty} \frac{d^{4} k}{(2 \pi)^{4}} \frac{d^{4} q}{(2 \pi)^{4}} R_{S}^{c t}(k, q ; p) \\
& =\int_{-\infty}^{\infty} \frac{d^{4} k}{(2 \pi)^{4}} \frac{d^{4} q}{(2 \pi)^{4}}\left\{D^{-1}(k) D^{-1}(q)^{-1} D^{-1}(p+k+q)\right. \\
& \left.-D^{-1}(k) D^{-1}(q) D^{-1}(k+q)\left(1-p^{2} D^{-1}(k+q)+4 \sum_{\mu} p_{\mu}^{2}(k+q)_{\mu}^{2} D^{-2}(k+q)\right)\right\}
\end{aligned}
$$

with the inverse continuum propagator

$$
D(p)=m^{2}+p^{2}=m^{2}+\sum_{\mu=1}^{4} p_{\mu}^{2} .
$$

We employ dimensional regularization for the evaluation of the Feynman integral (11) which can then be simplified according to

$$
\begin{aligned}
& \int_{-\infty}^{\infty} \frac{d^{d} k}{(2 \pi)^{d}} \frac{d^{d} q}{(2 \pi)^{d}} R_{S}^{c t}(k, q ; p) \\
& =\int_{-\infty}^{\infty} \frac{d^{d} k}{(2 \pi)^{d}} \frac{d^{d} q}{(2 \pi)^{d}}\left\{D^{-1}(k) D^{-1}(q)^{-1} D^{-1}(p+k+q)\right. \\
& \left.-D^{-1}(k) D^{-1}(q) D^{-1}(k+q)\left(1-p^{2} m^{2} D^{-2}(k+q)+\frac{\epsilon}{4} p^{2} D^{-1}(k+q)\right)\right\}+\ldots,
\end{aligned}
$$

where $\epsilon=4-d$ and we have neglected finite terms. One verifies in a straightforward manner that the divergent pieces of the single terms in Eq. (13) indeed cancel. This is most conveniently done by noting that in dimensional regularization the divergent pieces of the sunset integral with two different masses,

$$
I\left(m^{2}, m^{2}, M^{2} ; p^{2}\right)=\int_{-\infty}^{\infty} \frac{d^{d} k}{(2 \pi)^{d}} \frac{d^{d} q}{(2 \pi)^{d}} \frac{1}{\left[m^{2}+k^{2}\right]\left[m^{2}+q^{2}\right]\left[M^{2}+(k+q+p)^{2}\right]},
$$

are given by

$$
I_{\text {div }}\left(m^{2}, m^{2}, M^{2} ; p^{2}\right)=\frac{1}{\epsilon-1} \frac{\Gamma(\epsilon)}{(4 \pi)^{4-\epsilon}}\left[2 m^{2}\left(\frac{2}{\epsilon}+1-2 \ln m^{2}\right)+M^{2}\left(\frac{2}{\epsilon}+1-2 \ln M^{2}\right)+\frac{p^{2}}{2}\right] .
$$

The divergences of the scalar integrals in Eq. (13) can now be deduced directly from the expression in Eq. (15) and its differentiation with respect to $M^{2}$. One obtains

$$
\left.\int_{-\infty}^{\infty} \frac{d^{d} k}{(2 \pi)^{d}} \frac{d^{d} q}{(2 \pi)^{d}} R_{S}^{c t}(k, q ; p)\right|_{d i v}=0
$$

We have thus confirmed explicitly that the would-be divergences of the original lattice integral (1) are contained in the expression $t_{S}^{2} I_{S}$ of Eq. (10). As there is no external momentum left in the propagators of $t_{S}^{2} I_{S}$, one can restrict oneself in the following to loop integrals without external momenta in the propagators. 


\section{Coordinate space method}

After having applied the BPHZ formalism to the original sunset integral we now continue with the evaluation of the three lattice integrals appearing in $\int t_{S}^{2} I_{S}$, Eq. (10). Instead of calculating the divergent pieces in momentum space, it is more convenient to work in coordinate space. To this end, one can employ the results for the massive Green's function presented in [2]. We start with the integral

$$
\int_{-\pi}^{\pi} \frac{d^{4} k}{(2 \pi)^{4}} \frac{d^{4} q}{(2 \pi)^{4}} \frac{1}{\Delta(k) \Delta(q) \Delta(k+q)}=\sum_{x \in \Lambda} G(x)^{3}
$$

where $G$ is the lattice Green's function

$$
G(x)=\int_{-\pi}^{\pi} \frac{d^{4} k}{(2 \pi)^{4}} \frac{e^{i k \cdot x}}{\hat{k}^{2}+m^{2}} .
$$

In lattice units the sum over $G^{3}$ is finite and can be carried out numerically (with increasing precision for larger values of $m$ ) utilizing the precise numerical knowledge of the Green's function [2]. In order to isolate the would-be divergences for $a \rightarrow 0$, however, one recalls that in physical units the sunset diagram in Eq. (1) enters with a prefactor $1 / a^{2}$, where $a$ is the lattice spacing and the physical mass $\mu$ is given by $m=a \mu$. For large enough $x$ the first term in the small mass expansion of $G$, i.e. the massless Green's function $G_{m=0}$, behaves asymptotically as $1 / x^{2}$ and, hence, $G_{m=0}^{3}$ as $1 / x^{6}[1]$. One can thus perform the sum explicitly. But for the logarithmic divergences which arise in the subleading mass term one observes an asymptotic $1 / x^{4}$ behavior of the expansion coefficient of $G^{3}$ and the summation over all lattice sites cannot be performed numerically. We therefore proceed by splitting the sum of the Green's functions as follows:

$$
\sum_{x \in \Lambda} G(x)^{3}=G(0)^{3}+\sum_{x \neq 0}\left(G(x)^{3}-G_{a s}^{(0)}(x)^{3}\right)+\sum_{x \neq 0} G_{a s}^{(0)}(x)^{3} .
$$

In this expression, we have introduced the continuum Green's function

$$
G_{a s}^{(0)}(x)=\frac{1}{4 \pi^{2} x^{2}} m|x| K_{1}(m|x|)
$$

where $|x|=\sqrt{x^{2}}$ and $K_{1}$ represents the modified Bessel function of the second kind

$$
K_{\nu}(z)=\frac{\pi}{2} \frac{I_{-\nu}(z)-I_{\nu}(z)}{\sin (\nu \pi)}, \quad I_{\nu}(z)=\sum_{i=0}^{\infty} \frac{(z / 2)^{\nu+2 i}}{i ! \Gamma(\nu+i+1)} .
$$

The Green's function at the origin, $G(0)$, has been separated from the sum, as $G_{a s}^{(0)}(x)$ diverges at the origin. Note that we have merely subtracted (and added) the continuum Green's function which is sufficient to expand the difference in the first sum on the right side of Eq. (19) up to order $m^{2}$. In order to be able to expand to higher orders in $m^{2}$ one must subtract further asymptotic pieces of the lattice Green's function which are suppressed by increasing powers in the lattice spacing and represent corrections to the continuum expression. They have already been presented in [6], where the asymptotic behavior of the lattice Green's function for large $x$ has been studied. For completeness, we present in Appendix A an alternative and much simpler derivation of the asymptotic form of the lattice Green's function. 
For large $x$ and $m=0$ the difference $G(x)^{3}-G_{a s}^{(0)}(x)^{3}$ vanishes as $1 / x^{8}$ so that the small mass expansion can be performed up to $m^{2}$. To this end, we note that the small mass expansion of the Green's function is given by [2]

$$
G(x)=\sum_{i=0}^{\infty} a_{i}(x) m^{2 i}+m^{2} \ln \left(m^{2}\right) \sum_{i=0}^{\infty} b_{i}(x) m^{2 i},
$$

which converges absolutely for $m<2$. The functions $a_{i}(x)$ can be calculated to high accuracy using a set of recursion relations, while the $b_{i}(x)$ can be given in closed form

$$
b_{0}(x)=\frac{1}{16 \pi^{2}}, \quad b_{1}(x)=\frac{1}{128 \pi^{2}}\left(x^{2}-1\right), \quad b_{2}(x)=\frac{1}{3072 \pi^{2}}\left(\left(x^{2}\right)^{2}-4 x^{2}+3\right), \quad \text { etc. }
$$

Moreover, the asymptotic large- $x$ behavior of the coefficients $a_{i}, a_{i}^{a s}$, can be derived by expand$\operatorname{ing} G_{a s}^{(0)}$ in $m$

$$
\begin{aligned}
& a_{0}^{a s}(x)=\frac{1}{4 \pi^{2} x^{2}}+\mathcal{O}\left(1 / x^{4}\right) \\
& a_{1}^{a s}(x)=\frac{1}{16 \pi^{2}}\left[-1+2\left(\gamma_{E}+\ln \left(\frac{|x|}{2}\right)\right)\right]+\mathcal{O}\left(1 / x^{2}\right)
\end{aligned}
$$

with $\gamma_{E}=0.5772 \ldots$ being the Euler-Mascheroni constant. (In Eq. (24), subleading orders in $1 / x^{2}$ can be deduced from the higher order corrections of the asymptotic Green's function.) We therefore obtain up-to-and-including order $m^{2}$ the expressions

$$
G(0)^{3}=a_{0}(0)^{3}+3 m^{2} a_{0}(0)^{2} a_{1}(0)+3 m^{2} \ln \left(m^{2}\right) a_{0}(0)^{2} b_{0}(0)+\mathcal{O}\left(m^{4}\right)
$$

and

$$
\begin{aligned}
\sum_{x \neq 0}\left(G(x)^{3}-G_{a s}^{(0)}(x)^{3}\right)= & \sum_{x \neq 0}\left(a_{0}(x)^{3}-a_{0}^{a s}(x)^{3}\right)+3 m^{2} \sum_{x \neq 0}\left(a_{0}(x)^{2} a_{1}(x)-a_{0}^{a s}(x)^{2} a_{1}^{a s}(x)\right) \\
& +3 m^{2} \ln \left(m^{2}\right) \sum_{x \neq 0}\left(a_{0}(x)^{2} b_{0}(x)-a_{0}^{a s}(x)^{2} b_{0}(x)\right)+\mathcal{O}\left(m^{4}\right) \\
= & \sum_{x \neq 0}\left(a_{0}(x)^{3}-\frac{1}{64 \pi^{6} x^{6}}\right) \\
& +3 m^{2} \sum_{x \neq 0}\left(a_{0}(x)^{2} a_{1}(x)-\frac{1}{256 \pi^{6} x^{4}}\left[2 \gamma_{E}+2 \ln \left(\frac{|x|}{2}\right)-1\right]\right) \\
& +3 m^{2} \ln \left(m^{2}\right) \sum_{x \neq 0}\left(a_{0}(x)^{2} b_{0}(x)-\frac{1}{256 \pi^{6} x^{4}}\right)+\mathcal{O}\left(m^{4}\right) .
\end{aligned}
$$

In the continuum limit, the first and third sum in Eq. (26) lead to quadratic and logarithmic divergences, respectively, whereas the second sum remains finite.

We can now turn our attention to the evaluation of the last term in Eq. (19)

$$
\sum_{x \neq 0} G_{a s}^{(0)}(x)^{3}=\sum_{x \neq 0}\left(\frac{1}{4 \pi^{2} x^{2}} m|x| K_{1}(m|x|)\right)^{3}
$$


which we rewrite as

$$
\sum_{x \neq 0} G_{a s}^{(0)}(x)^{3}=\sum_{x \neq 0}\left[\left(\frac{1}{4 \pi^{2} x^{2}} m|x| K_{1}(m|x|)\right)^{3}-A(m, x, 4)\right]+\sum_{x \neq 0} A(m, x, 4) .
$$

Here, $A(m, x, d)$ is given by

$$
\begin{aligned}
A(m, x, d) & =\sum_{j=0}^{3} \kappa_{j} C\left(3\left[\frac{d}{2}-1\right]-j, m x\right)+\sum_{j=0}^{2} \kappa_{j+4} C\left(2\left[\frac{d}{2}-1\right]-j, m x\right) \\
& +\sum_{j=0}^{1} \kappa_{j+7} C\left(\frac{d}{2}-1-j, m x\right),
\end{aligned}
$$

with

$$
C(\nu, x)=\left(\frac{2}{x}\right)^{\nu} K_{\nu}(x)
$$

and the coefficients $\kappa_{0}, \ldots, \kappa_{8}$, which also depend on $m$ and $d$, are given in Appendix B. The function $A$ has been chosen in such a way that the difference

$$
b=\lim _{x \rightarrow 0}\left[\left(\frac{1}{4 \pi^{2} x^{2}} m|x| K_{1}(m|x|)\right)^{3}-A(m, x, 4)\right]=\mathcal{O}\left(m^{4}\right)
$$

remains finite at the origin. We can thus replace the first sum in Eq. (28) by the sum over all lattice sites including $x=0$. We are left with the evaluation of the difference on the right-hand side of Eq. (28) which is rewritten as

$$
\sum_{x \in \Lambda}\left[\left(\frac{1}{4 \pi^{2} x^{2}} m|x| K_{1}(m|x|)\right)^{3}-A(m, x, 4)\right]-b .
$$

Taking in Eq. (32) the Poisson resummation

$$
\sum_{x \in \Lambda} f(x)=\sum_{x \in \Lambda} \int_{-\infty}^{\infty} \frac{d^{4} p}{(2 \pi)^{4}} e^{-i p \cdot x} \tilde{f}(p)=\sum_{k} \tilde{f}(2 \pi k)
$$

yields

$$
\begin{aligned}
\sum_{x \neq 0} G_{a s}^{(0)}(x)^{3}= & \sum_{k} \lim _{d \rightarrow 4}\left[I\left(m^{2}, m^{2}, m^{2} ;(2 \pi k)^{2}\right)-\tilde{A}(m, 2 \pi k, d)\right]-b \\
& +\sum_{x \neq 0} A(m, x, 4)+\mathcal{O}\left(m^{4}\right)
\end{aligned}
$$

with $I$ the continuum sunset integral in $d$-dimensional space defined in Eq. (14) and with external momentum $2 \pi k$, while $\tilde{A}$ is given by

$$
\begin{aligned}
\tilde{A}(m, p, d)= & \frac{(4 \pi)^{d / 2}}{2}\left[\sum_{j=0}^{3} \kappa_{j} \Gamma(3+j-d) \frac{m^{6+2 j-3 d}}{\left(p^{2}+m^{2}\right)^{3+j-d}}+\sum_{j=0}^{2} \kappa_{j+4} \Gamma\left(2+j-\frac{d}{2}\right)\right. \\
& \left.\times \frac{m^{4+2 j-2 d}}{\left(p^{2}+m^{2}\right)^{2+j-\frac{d}{2}}}+\sum_{j=0}^{1} \kappa_{j+7} \Gamma(1+j) \frac{m^{2+2 j-d}}{\left(p^{2}+m^{2}\right)^{1+j}}\right] .
\end{aligned}
$$


We have analytically continued the expressions in the first sum of Eq. (34) to $d$-dimensional space. For $d=4$, the single terms in the difference of Eq. (28) diverge at the origin and cannot be calculated separately, whereas for $d$ small enough both terms remain finite and can be evaluated. Taking afterwards the $d \rightarrow 4$ limit one confirms that the divergences in $d$ cancel out in the difference. By continuing to $d$ dimensions we have automatically introduced dimensionally regularized integrals. In the course of evaluating sunset diagrams with momenta $2 \pi k$ in dimensional regularization it is more convenient to discuss the case with momentum $k=0$ separately. The results for external momentum $k=0$ are presented, e.g., in $[7,8]$

$$
\begin{aligned}
I\left(m^{2}, m^{2}, m^{2} ; 0\right) & =-\frac{3 m^{2}}{128 \pi^{4}} \frac{1}{(d-4)^{2}}+\frac{3 m^{2}}{256 \pi^{4}}\left(3-2 \gamma_{E}-2 \ln \left(m^{2}\right)+2 \ln (4 \pi)\right) \frac{1}{d-4} \\
& +\frac{m^{2}}{256 \pi^{4}}\left[-\frac{21}{2}-3 \gamma_{E}^{2}-\frac{\pi^{2}}{4}-9 \ln (4 \pi)-3 \ln ^{2}(4 \pi)+2 \sqrt{3} \mathrm{Cl}_{2}\left(\frac{\pi}{3}\right)\right. \\
& \left.+\gamma_{E}(9+6 \ln (4 \pi))+\ln \left(m^{2}\right)\left(9-6 \gamma_{E}+6 \ln (4 \pi)\right)-3 \ln ^{2}\left(m^{2}\right)\right]
\end{aligned}
$$

with $\mathrm{Cl}_{2}$ being the Clausen function. For $k \neq 0$, on the other hand, one can expand in powers of $m^{2} /(2 \pi k)^{2}[7,8]$

$$
\begin{aligned}
I\left(m^{2}, m^{2}, m^{2} ; p^{2}\right) & =-\frac{3 m^{2}}{128 \pi^{4}} \frac{1}{(d-4)^{2}}+\frac{p^{2}-12 m^{2} \ln \left(m^{2}\right)+6 m^{2}\left(3-2 \gamma_{E}+2 \ln (4 \pi)\right)}{512 \pi^{4}} \frac{1}{d-4} \\
& +\frac{p^{2}}{2048 \pi^{4}}\left[-13+4 \gamma_{E}-4 \ln (4 \pi)+4 \ln \left(p^{2}\right)\right]-\frac{m^{2}}{1024 \pi^{4}}\left[30+12 \gamma_{E}\left(-3+\gamma_{E}\right)\right. \\
& +\pi^{2}+36 \ln (4 \pi)+6\left[\ln ^{2}\left(m^{2}\right)-\ln ^{2}\left(p^{2}\right)+2 \ln \left(m^{2}\right)\left(-3+2 \gamma_{E}+\ln \left(p^{2}\right)\right.\right. \\
& \left.\left.-2 \ln (4 \pi))+2 \ln (4 \pi)\left(-2 \gamma_{E}+\ln (4 \pi)\right)\right]\right]+m^{2} \mathcal{O}\left(m^{2} / p^{2}\right)
\end{aligned}
$$

For our purposes we only need to expand up to $m^{2}$. Note that in the difference $I-\tilde{A}$ the $1 /(d-4)$ divergences cancel and the difference $I-\tilde{A}$ scales like $1 / k^{6}$ for large $k$ so that the sum over $k$ converges.

We are left with the evaluation of the sum $\sum_{x \neq 0} A(m, x, 4)$ in Eq. (34). To this aim, it is convenient to work with the integral representation of the Bessel functions $K$ leading to, cf. Eq. (30),

$$
C(\nu, m x)=\frac{1}{2 m^{2 \nu}} \int_{0}^{\infty} d \lambda \lambda^{-\nu-1} \exp \left(-m^{2} \lambda-\frac{x^{2}}{4 \lambda}\right) .
$$

Since the function $A$ is a linear combination of the $C$ functions, it is sufficient to demonstrate the calculation for the sum

$$
\sum_{x \neq 0} C(\nu, m x)
$$

or in more general - if we are interested in the calculation of $m^{4}$ and higher order correctionsterms of the form

$$
\sum_{x \neq 0} h_{D}(x) C(\nu, m x)
$$

Here, $h_{D}(x)$ is a harmonic homogeneous polynomial of degree $\mathrm{D}$ with the leading orders given by

$$
h_{0}(x)=1, \quad h_{4}(x)=2 x^{4}-\left(x^{2}\right)^{2}, \quad h_{6}(x)=16 x^{6}-20 x^{2} x^{4}+5\left(x^{2}\right)^{3}, \quad \ldots \quad .
$$


Following [1] we introduce the heat kernel

$$
k\left(\lambda, h_{D}\right)=\sum_{x} h_{D}(x) e^{-\pi \lambda x^{2}}
$$

and rewrite the original sum in the form

$$
\sum_{x \neq 0} h_{D}(x) C(\nu, m x)=\frac{1}{2}\left(\frac{4 \pi}{m^{2}}\right)^{\nu} \int_{0}^{\infty} d \lambda \lambda^{-\nu-1} e^{-\frac{m^{2}}{4 \pi} \lambda}\left[k\left(1 / \lambda, h_{D}\right)-h_{D}(0)\right] .
$$

Poisson resummation and the fact that $h_{D}(x)$ is a harmonic homogeneous polynomial of degree $D$ lead to the relation

$$
k\left(\lambda, h_{D}\right)=(-1)^{D / 2} \lambda^{-D-2} k\left(1 / \lambda, h_{D}\right) .
$$

Employing this relation we can separate the regular and singular parts of the integral

$$
\begin{aligned}
\sum_{x \neq 0} h_{D}(x) C(\nu, m x) & =\frac{1}{2}\left(\frac{4 \pi}{m^{2}}\right)^{\nu} \int_{1}^{\infty} d \lambda\left[k\left(\lambda, h_{D}\right)-h_{D}(0)\right]\left[(-1)^{D / 2} e^{-\frac{m^{2}}{4 \pi} \lambda} \lambda^{D-\nu+1}+e^{-\frac{m^{2}}{4 \pi \lambda}} \lambda^{\nu-1}\right] \\
& +\frac{1}{2}\left(\frac{4 \pi}{m^{2}}\right)^{\nu} h_{D}(0)\left[(-1)^{D / 2} E_{\nu-D-1}\left(\frac{m^{2}}{4 \pi}\right)-E_{\nu+1}\left(\frac{m^{2}}{4 \pi}\right)\right],
\end{aligned}
$$

with the exponential integral functions $E_{n}$,

$$
E_{n}(z)=\int_{1}^{\infty} d \lambda e^{-z \lambda} \lambda^{-n}
$$

The first term in Eq. (45) is finite for all values of $\nu$ and can be Taylor expanded in $m^{2}$, while the exponential integral functions entail the logarithmic terms. The sum over the $A$ functions can now be represented as a linear combination of regularized zeta functions and their derivatives with respect to $s$

$$
\xi_{R}\left(s, h_{D}\right)=\int_{1}^{\infty} d \lambda\left[\lambda^{s-1}+(-1)^{D / 2} \lambda^{D-s+1}\right]\left[k(\lambda, h)-h_{D}(0)\right], \quad \xi_{R}^{(i)}\left(s, h_{D}\right)=\frac{d^{i}}{d s^{i}} \xi_{R}\left(s, h_{D}\right) .
$$

The regularized zeta functions are related to the generalized zeta functions via

$$
\xi\left(s, h_{D}\right)=\sum_{x \neq 0} h_{D}(x)\left(x^{2}\right)^{-s}=\frac{\pi^{s}}{\Gamma(s)}\left[\frac{2 h_{D}(0)}{s(s-2)}+\xi_{R}\left(s, h_{D}\right)\right] .
$$

Altogether, we obtain

$$
\begin{aligned}
\sum_{x \neq 0} A(m, x, 4) & =\frac{1}{(4 \pi)^{3}}\left[\frac{1}{3}+\frac{\xi_{R}\left(3, h_{0}\right)}{2}\right]+m^{2} \frac{1}{(4 \pi)^{4}}\left[\frac{11}{6}-\frac{3 \gamma_{E}}{2}-\frac{3 \gamma_{E}^{2}}{2}+\frac{\pi^{2}}{4}+\frac{3}{2} \ln (4 \pi)\right. \\
& \left.+3 \gamma_{E} \ln (4 \pi)-\frac{3}{2} \ln ^{2}(4 \pi)+3 \gamma_{E} \xi_{R}\left(2, h_{0}\right)-3 \ln (4 \pi) \xi_{R}\left(2, h_{0}\right)-3 \xi_{R}^{(1)}\left(2, h_{0}\right)\right] \\
& +m^{2} \ln \left(m^{2}\right) \frac{1}{(4 \pi)^{4}}\left[-\frac{3}{2}-3 \gamma_{E}+3 \ln (4 \pi)+3 \xi_{R}\left(2, h_{0}\right)\right]-m^{2} \ln ^{2}\left(m^{2}\right) \frac{3}{2} \frac{1}{(4 \pi)^{4}} \\
& +\mathcal{O}\left(m^{4}\right) .
\end{aligned}
$$


We can now summarize the quadratic, logarithmic and bilogarithmic divergences as well as the finite part of the integral in Eq. (17). From Eqs. (25), (26),(36),(37)and (49) we obtain the quadratic would-be divergences

$$
\begin{aligned}
& \left.\int_{-\pi}^{\pi} \frac{d^{4} k}{(2 \pi)^{4}} \frac{d^{4} q}{(2 \pi)^{4}} \frac{1}{\Delta(k) \Delta(q) \Delta(k+q)}\right|_{\text {quadr.div. }} \\
& =a_{0}(0)^{3}+\sum_{x \neq 0}\left(a_{0}(x)^{3}-\frac{1}{64 \pi^{6} x^{6}}\right)+\frac{1}{(4 \pi)^{3}}\left[\frac{1}{3}+\frac{\xi_{R}\left(3, h_{0}\right)}{2}\right]
\end{aligned}
$$

the logarithmic divergences

$$
\begin{aligned}
& \left.\int_{-\pi}^{\pi} \frac{d^{4} k}{(2 \pi)^{4}} \frac{d^{4} q}{(2 \pi)^{4}} \frac{1}{\Delta(k) \Delta(q) \Delta(k+q)}\right|_{\text {log.div. }} \\
& =m^{2} \ln \left(m^{2}\right)\left[3 a_{0}(0)^{2} b_{0}(0)+\sum_{x \neq 0}\left(3 a_{0}(x)^{2} b_{0}(x)-\frac{3}{256 \pi^{6} x^{4}}\right)+\frac{1}{(4 \pi)^{4}}\left(-\frac{3}{2}-3 \gamma_{E}\right.\right. \\
& \left.\left.+3 \ln (4 \pi)+3 \xi_{R}\left(2, h_{0}\right)\right)\right]
\end{aligned}
$$

the bilogarithmic divergences

$$
\left.\int_{-\pi}^{\pi} \frac{d^{4} k}{(2 \pi)^{4}} \frac{d^{4} q}{(2 \pi)^{4}} \frac{1}{\Delta(k) \Delta(q) \Delta(k+q)}\right|_{\text {bilog.div. }}=-\frac{3}{2(4 \pi)^{4}} m^{2} \ln ^{2}\left(m^{2}\right),
$$

as well as the finite remainder in the continuum limit

$$
\begin{aligned}
& \left.\int_{-\pi}^{\pi} \frac{d^{4} k}{(2 \pi)^{4}} \frac{d^{4} q}{(2 \pi)^{4}} \frac{1}{\Delta(k) \Delta(q) \Delta(k+q)}\right|_{\text {finite }} \\
& =m^{2}\left[3 a_{0}(0)^{2} a_{1}(0)+\sum_{x \neq 0}\left(3 a_{0}(x)^{2} a_{1}(x)-\frac{3}{256 \pi^{6} x^{4}}\left(2 \gamma_{E}+2 \ln (x / 2)-1\right)\right)+\frac{1}{(4 \pi)^{4}}\left(-\frac{15}{4}\right.\right. \\
& -\frac{3 \gamma_{E}}{2}-\frac{3 \gamma_{E}^{2}}{2}+\frac{\pi^{2}}{4}+2 \sqrt{3} \mathrm{Cl}_{2}\left(\frac{\pi}{3}\right)+\frac{3}{2} \ln (4 \pi)+3 \gamma_{E} \ln (4 \pi)-\frac{3}{2} \ln ^{2}(4 \pi)+3 \gamma_{E} \xi_{R}\left(2, h_{0}\right) \\
& \left.\left.-3 \ln (4 \pi) \xi_{R}\left(2, h_{0}\right)-3 \xi_{R}^{(1)}\left(2, h_{0}\right)\right)\right]
\end{aligned}
$$

So far, we have evaluated the divergent components of the sunset integral given in Eq. (17). The calculation of the divergences in the second integrand in $t_{S}^{2} I_{S}$, Eq. (10), is immediately obtained via

$$
\int_{-\pi}^{\pi} \frac{d^{4} k}{(2 \pi)^{4}} \frac{d^{4} q}{(2 \pi)^{4}} \frac{1}{\Delta(k) \Delta(q) \Delta(k+q)^{2}}=-\frac{1}{3} \frac{d}{d m^{2}} \int_{-\pi}^{\pi} \frac{d^{4} k}{(2 \pi)^{4}} \frac{d^{4} q}{(2 \pi)^{4}} \frac{1}{\Delta(k) \Delta(q) \Delta(k+q)}
$$

and by noting that this time we only need the small mass expansion at leading order due to the presence of the prefactor $\bar{p}^{2}$ in Eq. (10). Thus, the second term in $t_{S}^{2} I_{S}$ contributes only to the logarithmic divergences.

The third and final contribution in $t_{S}^{2} I_{S}$, Eq. (10), is given by

$$
4 \int_{-\pi}^{\pi} \frac{d^{4} k}{(2 \pi)^{4}} \frac{d^{4} q}{(2 \pi)^{4}} \frac{\sin ^{2}\left(k_{\mu}+q_{\mu}\right)}{\Delta(k) \Delta(q) \Delta(k+q)^{3}}=-\frac{1}{4} \sum_{x} G_{1}(x)^{2} \sum_{\mu}\left[\nabla_{\mu}+\nabla_{\mu}^{*}\right]^{2} G_{3}(x) .
$$


Here, we have introduced the notation

$$
G_{i}(x)=\int_{-\pi}^{\pi} \frac{d^{4} k}{(2 \pi)^{4}} \frac{e^{i k \cdot x}}{[\Delta(k)]^{i}}
$$

and the backward and forward lattice derivatives are defined by

$$
\nabla_{\mu}^{*} f(x)=f(x)-f(x-\mu), \quad \nabla_{\mu} f(x)=f(x+\mu)-f(x) .
$$

One easily verifies that the most general lattice integral with one mass arising in the BPHZ subtraction procedure has the form

$$
\int_{-\pi}^{\pi} \frac{d^{4} k}{(2 \pi)^{4}} \frac{d^{4} q}{(2 \pi)^{4}} \frac{\prod_{\mu} \hat{k}_{\mu}^{2 l_{\mu}^{1}} \hat{q}_{\mu}^{2 l_{\mu}^{2}}\left(\widehat{k+q}_{\mu}^{2 l_{\mu}^{3}}\right.}{\Delta(k)^{\alpha} \Delta(q)^{\beta} \Delta(k+q)^{\gamma}} .
$$

From Eq. (55) it is obvious how a generic integral of the form (58) can be treated with the proposed coordinate space method. The presented approach is therefore suited to evaluate any two-loop integral without external momentum which arises in the BPHZ procedure. The extension of this method to scalar field theories with different masses is also straightforward.

We proceed as above by separating in Eq. (55) the contribution at the origin from the remaining sum and isolating the corresponding continuum result

$$
\begin{aligned}
& -\frac{1}{4} \sum_{x} G_{1}(x)^{2} \sum_{\mu}\left[\nabla_{\mu}+\nabla_{\mu}^{*}\right]^{2} G_{3}(x)=-\frac{1}{4} G_{1}(0)^{2} \sum_{\mu}\left[\nabla_{\mu}+\nabla_{\mu}^{*}\right]^{2} G_{3}(0)-\sum_{x \neq 0}\left[\frac{1}{4} G_{1}(x)^{2}\right. \\
& \left.\times \sum_{\mu}\left[\nabla_{\mu}+\nabla_{\mu}^{*}\right]^{2} G_{3}(x)-G_{1}^{c}(x)^{2} \sum_{\mu}\left(\frac{\partial}{\partial x_{\mu}}\right)^{2} G_{3}^{c}(x)\right]-\sum_{x \neq 0} G_{1}^{c}(x)^{2} \sum_{\mu}\left(\frac{\partial}{\partial x_{\mu}}\right)^{2} G_{3}^{c}(x)
\end{aligned}
$$

with

$$
G_{i}^{c}(x)=\int_{-\infty}^{\infty} \frac{d^{4} k}{(2 \pi)^{4}} \frac{e^{i k \cdot x}}{[D(k)]^{i}} .
$$

(Note that in our notation $G_{1}^{c}=G_{a s}^{(0)}$.) The first term on the right side of Eq. (59) can be directly derived from the Green's function at the origin [2], and the subsequent term can also be calculated numerically, since the difference of functions in the sum behaves like $1 / x^{6}$ for large $x$ in the $m \rightarrow 0$ limit. In contrast, the rightmost term in Eq. (59) must be further decomposed

$$
\begin{aligned}
& \sum_{x \neq 0} G_{1}^{c}(x)^{2} \sum_{\mu}\left(\frac{\partial}{\partial x_{\mu}}\right)^{2} G_{3}^{c}(x) \\
& =\sum_{x}\left[G_{1}^{c}(x)^{2} \sum_{\mu}\left(\frac{\partial}{\partial x_{\mu}}\right)^{2} G_{3}^{c}(x)-B(m, x, 4)\right] \\
& -\lim _{x \rightarrow 0}\left(G_{1}^{c}(x)^{2} \sum_{\mu}\left(\frac{\partial}{\partial x_{\mu}}\right)^{2} G_{3}^{c}(x)-B(m, x, 4)\right)+\sum_{x \neq 0} B(m, x, 4) \\
& =\sum_{k} \int d^{4} x e^{i 2 \pi k \cdot x}\left[G_{1}^{c}(x)^{2} \sum_{\mu}\left(\frac{\partial}{\partial x_{\mu}}\right)^{2} G_{3}^{c}(x)-B(m, x, 4)\right] \\
& -\lim _{x \rightarrow 0}\left(G_{1}^{c}(x)^{2} \sum_{\mu}\left(\frac{\partial}{\partial x_{\mu}}\right)^{2} G_{3}^{c}(x)-B(m, x, 4)\right)+\sum_{x \neq 0} B(m, x, 4),
\end{aligned}
$$


where the function $B(m, x, d)$ is defined as

$$
\begin{aligned}
B(m, x, d) & =\sum_{j=0}^{3} \lambda_{j} C\left(3\left[\frac{d}{2}-1\right]-j, m x\right)+\sum_{j=0}^{2} \lambda_{j+4} C\left(2\left[\frac{d}{2}-1\right]-j, m x\right) \\
& +\sum_{j=0}^{1} \lambda_{j+7} C\left(\frac{d}{2}-1-j, m x\right)
\end{aligned}
$$

with coefficients $\lambda_{j}$, which also depend on $m$ and $d$, given in Appendix B. The function $B(m, x, d)$ has been introduced in order to apply the Poisson summation formula. The difference at the origin $x=0$ in Eq. (61) is given by

$$
\lim _{x \rightarrow 0}\left(G_{1}^{c}(x)^{2} \sum_{\mu}\left(\frac{\partial}{\partial x_{\mu}}\right)^{2} G_{3}^{c}(x)-B(m, x, 4)\right)=\mathcal{O}\left(m^{4}\right) .
$$

For the evaluation of the first term in Eq. (61), on the other hand, we utilize

$$
\begin{aligned}
& \int d^{d} x e^{i p \cdot x} G_{1}^{c}(x)^{2} \sum_{\mu}\left(\frac{\partial}{\partial x_{\mu}}\right)^{2} G_{3}^{c}(x)=\int d^{d} x e^{i p \cdot x} G_{1}^{c}(x)^{2}\left(\frac{d}{4} \frac{\partial}{\partial m^{2}}+\frac{x^{2}}{8}\right) G_{1}^{c}(x) \\
& =\left[\frac{d}{12} \frac{\partial}{\partial m^{2}}-\frac{1}{8}\left(\frac{\partial^{2}}{\partial|p|^{2}}+\frac{d-1}{|p|} \frac{\partial}{\partial|p|}\right)\right] \int d^{d} x e^{i p \cdot x} G_{1}^{c}(x)^{3},
\end{aligned}
$$

where we introduced analytical continuation to $d$ dimensions in order to evaluate both terms in the first sum of Eq. (61) separately. For $k \neq 0$ we can directly apply the differential operators of the last equation to the result of Eq. (37). For $k=0$, on the other hand, we apply the differential operators to the small $p$ expansion of the continuum sunset diagram. The leading order of this expansion has already been presented in Eq. (36), while the $p^{2}$ terms are given by

$$
p^{2}\left[\frac{1}{512(-4+d) \pi^{4}}+\frac{-27+36 \gamma_{E}+36 \ln \left(m^{2}\right)-36 \ln (4 \pi)+32 \sqrt{3} \mathrm{Cl}_{2}\left(\frac{\pi}{3}\right)}{18432 \pi^{4}}\right] .
$$

Note that for our considerations we can neglect $p^{4}$ and higher contributions. The Poisson resummation for $B$ follows immediately by noting that $B$ has the same structure as $A$ in Eq. (29) and replacing $\kappa_{i}$ by $\lambda_{i}$ in Eq. (35). As final result we obtain

$$
\int d^{4} x\left[G_{1}^{c}(x)^{2} \sum_{\mu}\left(\frac{\partial}{\partial x_{\mu}}\right)^{2} G_{3}^{c}(x)-B(m, x, 4)\right]=\frac{1}{(4 \pi)^{4}}\left[-\frac{9}{4}+\frac{2}{3 \sqrt{3}} \mathrm{Cl}_{2}\left(\frac{\pi}{3}\right)\right] .
$$

Summarizing the divergent and finite portions of the integral in Eq. (55) which remain in 
the continuum limit one obtains

$$
\begin{aligned}
& \left.\quad 4 \int_{-\pi}^{\pi} \frac{d^{4} k}{(2 \pi)^{4}} \frac{d^{4} q}{(2 \pi)^{4}} \frac{\sin ^{2}\left(k_{\mu}+q_{\mu}\right)}{\Delta(k) \Delta(q) \Delta(k+q)^{3}}\right|_{\text {log.div. }} \\
& =\ln \left(m^{2}\right)\left\{-\frac{1}{16 \pi^{2}} a_{0}(0)^{2}+\sum_{x \neq 0}\left[-\frac{1}{4} a_{0}(x)^{2} \sum_{\mu}\left[\nabla_{\mu}+\nabla_{\mu}^{*}\right]^{2} b_{1}(x)+\frac{1}{256 \pi^{6} x^{4}}\right]\right. \\
& \left.+\frac{1}{(4 \pi)^{4}}\left(2+\gamma_{E}-\ln (4 \pi)-\xi_{R}\left(2, h_{0}\right)\right)\right\}, \quad=\frac{1}{2(4 \pi)^{4}} \ln ^{2}\left(m^{2}\right), \\
& \left.\left.4 \int_{-\pi}^{\pi} \frac{d^{4} k}{(2 \pi)^{4}} \frac{d^{4} q}{(2 \pi)^{4}} \frac{\sin ^{2}\left(k_{\mu}+q_{\mu}\right)}{\Delta(k) \Delta(q) \Delta(k+q)^{3}}\right|_{\text {bilog.div. }} \frac{d^{4} k}{(2 \pi)^{4}} \frac{d^{4} q}{(2 \pi)^{4}} \frac{\sin ^{2}\left(k_{\mu}+q_{\mu}\right)}{\Delta(k) \Delta(q) \Delta(k+q)^{3}}\right|_{\text {finite }}=-a_{0}(0)^{2}\left[\frac{3}{32 \pi^{2}}+2\left(a_{2}(2,0,0,0)-a_{2}(0)\right)\right] \\
& +\sum_{x \neq 0}\left[\frac{1}{512 \pi^{6} x^{4}}\left(1+4 \gamma_{E}+4 \ln (x / 2)\right)-\frac{1}{4} a_{0}(x)^{2} \sum_{\mu}\left[\nabla_{\mu}+\nabla_{\mu}^{*}\right]^{2}\left(a_{2}(x)+\frac{3}{2} b_{1}(x)\right)\right] \\
& +\frac{1}{(4 \pi)^{4}}\left[\frac{5}{2}+2 \gamma_{E}+\frac{\gamma_{E}^{2}}{2}-\frac{\pi^{2}}{12}-\frac{2 \mathrm{Cl}_{2}\left(\frac{\pi}{3}\right)}{3 \sqrt{3}}-2 \ln (4 \pi)-\gamma_{E} \ln (4 \pi)+\frac{1}{2} \ln ^{2}(4 \pi)-\frac{3 \xi_{R}\left(2, h_{0}\right)}{2}\right. \\
& \left.-\gamma_{E} \xi_{R}\left(2, h_{0}\right)+\ln (4 \pi) \xi_{R}\left(2, h_{0}\right)+\xi_{R}^{(1)}\left(2, h_{0}\right)\right] .
\end{aligned}
$$

\section{Numerical results}

The Equations (50-53) and (66-68) can in principle be evaluated numerically. However, the respective sums converge slowly. In order to increase the precision of the numerical results one must therefore accelerate the convergence of the sums. This can be achieved, e.g., by using the zeta function technique introduced in [1].

The small mass expansions of the integrals in Eqs. (1) and (55) are given by

$$
\begin{aligned}
& \int_{-\pi}^{\pi} \frac{d^{4} k}{(2 \pi)^{4}} \frac{d^{4} q}{(2 \pi)^{4}} \frac{1}{\Delta(k) \Delta(q) \Delta(k+q)}=\sum_{i=0}^{\infty} c_{i} m^{2 i}+m^{2} \ln \left(m^{2}\right) \sum_{i=0}^{\infty} d_{i} m^{2 i}+m^{2} \ln ^{2}\left(m^{2}\right) \sum_{i=0}^{\infty} e_{i} m^{2 i}, \\
& 4 \int_{-\pi}^{\pi} \frac{d^{4} k}{(2 \pi)^{4}} \frac{d^{4} q}{(2 \pi)^{4}} \frac{\sin ^{2}\left(k_{\mu}+q_{\mu}\right)}{\Delta(k) \Delta(q) \Delta(k+q)^{3}}=\sum_{i=0}^{\infty} f_{i} m^{2 i}+\ln \left(m^{2}\right) \sum_{i=0}^{\infty} g_{i} m^{2 i}+\ln ^{2}\left(m^{2}\right) \sum_{i=0}^{\infty} h_{i} m^{2 i} .
\end{aligned}
$$

Recall that the expansion for the integral in Eq. (54) follows directly from the first equation above by differentiation with respect to $\mathrm{m}^{2}$. Utilizing the zeta function method we have calculated the first few coefficients of these series. We obtain

$$
\begin{array}{ll}
c_{0}=0.0040430548122 \ldots, & c_{1}=-0.0024114634124 \ldots, \\
d_{0}=0.0006968046967 \ldots, & e_{0}=-\frac{3}{2} \frac{1}{(4 \pi)^{4}}
\end{array}
$$

and

$$
f_{0}=0.0000731523655 \ldots, \quad g_{0}=-0.0001721159925 \ldots, \quad h_{0}=\frac{1}{2} \frac{1}{(4 \pi)^{4}} .
$$


In principle, we can calculate higher coefficients with this method, but for renormalization purposes this is not necessary. Also, the precision of the coefficients can easily be improved by including higher orders in the zeta function technique and increasing computing time a bit.

\section{Additional relations for logarithmic terms}

The logarithmic terms can, in fact, be related to one-loop tadpoles. Starting from the identity

$$
\sum_{x} e^{i p x} G(x)^{2}=\int_{-\pi}^{\pi} \frac{d^{4} k}{(2 \pi)^{4}} \frac{1}{\Delta(k) \Delta(p+k)} .
$$

one has for $p=0$

$$
\sum_{x} G(x)^{2}=-\frac{\partial}{\partial m^{2}} G(0)
$$

On the other hand, decomposition of the sum leads to

$$
\sum_{x} G(x)^{2}=a_{0}(0)^{2}+\sum_{x \neq 0}\left(a_{0}(x)^{2}-\frac{1}{16 \pi^{4} x^{4}}\right)+\sum_{x \neq 0} G_{a s}(x)^{2}+\mathcal{O}\left(m^{2}\right) .
$$

Employing the small mass expansion of the tadpole

$$
\frac{\partial}{\partial m^{2}} G(0)=a_{1}(0)+b_{0}(0)+b_{0}(0) \ln \left(m^{2}\right)+\mathcal{O}\left(m^{2}\right)
$$

one gets

$$
\sum_{x \neq 0}\left(a_{0}(x)^{2}-\frac{1}{16 \pi^{4} x^{4}}\right)=-a_{0}(0)^{2}-\sum_{x \neq 0} G_{a s}(x)^{2}-a_{1}(0)-b_{0}(0)-b_{0}(0) \ln \left(m^{2}\right)+\mathcal{O}\left(m^{2}\right) .
$$

The sum over the asymptotic Green's function on the right-hand side can be calculated with the method presented in Sec. 3:

$$
\sum_{x \neq 0} G_{a s}(x)^{2}=\frac{1}{(4 \pi)^{2}}\left[-\frac{5}{2}-\gamma_{E}-\ln \left(m^{2}\right)+\ln (4 \pi)+\xi_{R}\left(2, h_{0}\right)\right]+\mathcal{O}\left(m^{2}\right) .
$$

Comparison with Eq. (51) leads immediately to the logarithmic divergence

$$
\left.\int_{-\pi}^{\pi} \frac{d^{4} k}{(2 \pi)^{4}} \frac{d^{4} q}{(2 \pi)^{4}} \frac{1}{\Delta(k) \Delta(q) \Delta(k+q)}\right|_{\log . \text { div. }}=m^{2} \ln \left(m^{2}\right) \frac{3}{(4 \pi)^{4}}\left[1-(4 \pi)^{2} a_{1}(0)\right]
$$

which reproduces the value for $d_{0}$ given in Eq. (69) when using $a_{1}=-0.030345 \ldots$ [2]. By similar considerations we obtain for the logarithmic term of the tensor integral

$$
\left.4 \int_{-\pi}^{\pi} \frac{d^{4} k}{(2 \pi)^{4}} \frac{d^{4} q}{(2 \pi)^{4}} \frac{\sin ^{2}\left(k_{\mu}+q_{\mu}\right)}{\Delta(k) \Delta(q) \Delta(k+q)^{3}}\right|_{\text {log.div. }}=\ln \left(m^{2}\right) \frac{1}{(4 \pi)^{4}}\left[\frac{1}{2}+(4 \pi)^{2} a_{1}(0)\right]
$$

which again is in agreement with $g_{0}$ from Eq. (69). The additional relations derived here are not restricted to the leading order in the small mass expansion and one can obtain similar relations 
for higher order logarithmic pieces. In the remainder of this section, we will briefly illustrate the general strategy in relating higher order logarithmic terms of two-loop diagrams to one-loop tadpole integrals. For brevity, we will restrict ourselves to $\sum_{x} G(x)^{3}$, but the generalization to tensor integrals is straightforward. We decompose $\sum_{x} G(x)^{3}$ as

$$
\sum_{x} G(x)^{3}=G(0)^{3}+\sum_{x \neq 0}\left[G(x)^{3}-F(x)\right]+\sum_{x \neq 0} F(x)
$$

where the function $F(x)$ is chosen in such a way that the difference in the sum can be trivially expanded in the small mass up to a given order. The small mass expansion of $F(x)$ reads

$$
F(x)=\sum_{j=0}^{3} \sum_{i=0}^{\infty} f_{i}^{(j)}(x) m^{2 i}\left[m^{2} \ln \left(m^{2}\right)\right]^{j}
$$

with analytically known expansion coefficients $f_{i}^{(j)}$. For the $n$-th order logarithmic term of the difference we get

$$
\sum_{x \neq 0}\left[G(x)^{3}-F(x)\right]_{m^{2 n} \ln \left(m^{2}\right)}=m^{2 n} \ln \left(m^{2}\right) \sum_{x \neq 0}\left[\sum_{i+j+k=n-1} b_{i}(x) a_{j}(x) a_{k}(x)-f_{n-1}^{(1)}(x)\right] .
$$

On the other hand, one has

$$
\sum_{x} x^{2 i} G(x)^{2}=\delta_{i, 0} G(0)^{2}+\sum_{x \neq 0}\left[x^{2 i} G(x)^{2}-H(x)\right]+\sum_{x \neq 0} H(x),
$$

where again the function $H(x)$ is chosen in such a way that the difference in the sum can be expanded in the small mass up to a given order. If the small mass expansion of $H(x)$ is given by

$$
H(x)=\sum_{j=0}^{2} \sum_{i=0}^{\infty} h_{i}^{(j)}(x) m^{2 i}\left[m^{2} \ln \left(m^{2}\right)\right]^{j},
$$

then the $l$-th order of the difference reads

$$
\sum_{x \neq 0}\left[x^{2 i} G(x)^{2}-H(x)\right]_{m^{2 l}}=m^{2 l} \sum_{x \neq 0}\left[\sum_{j+k=l} x^{2 i} a_{j}(x) a_{k}(x)-h_{l}^{(0)}(x)\right] .
$$

Since $b_{i}(x)$ is a polynomial in $x$ we can always describe the lattice sum of the logarithmic term in Eq. (80) as a linear combination of the terms

$$
\sum_{x \neq 0}\left[x^{2 i} G(x)^{2}-H(x)\right]_{m^{2 l}}
$$

and get in this way relations between the logarithmic pieces of $\sum_{x} G(x)^{3}$ and terms of the type $\sum_{x} x^{2 i_{1}} \ldots x^{2 i_{n}} G(x)^{2}$. The latter terms can in turn be written as linear combinations of tadpoles with tensor structures

$$
\begin{aligned}
& \sum_{x} x^{2 i_{1}} \ldots x^{2 i_{n}} G(x)^{2} \\
& =\left.\left[(-1)^{i_{1}} \sum_{\mu}\left(\frac{\partial}{\partial p_{\mu}}\right)^{2 i_{1}}\right] \ldots\left[(-1)^{i_{n}} \sum_{\mu}\left(\frac{\partial}{\partial p_{\mu}}\right)^{2 i_{n}}\right]\right|_{p=0} \int_{-\pi}^{\pi} \frac{d^{4} k}{(2 \pi)^{4}} \frac{1}{\Delta(k)} \frac{1}{\Delta(p+k)}
\end{aligned}
$$


such that the logarithmic pieces in $\sum_{x} G(x)^{3}$ are expressed in terms of (tensor-)tadpole integrals.

\section{Conclusions}

In this work, we have extracted the logarithmic and quadratic divergences of the basic sunset diagram for a massive scalar field on the lattice and calculated the coefficients of its would-be divergences to very high precision. As a first step, the BPHZ fomalism has been applied to the sunset integral leading to three integrals without external momentum in the propagators. The quadratic and (bi-) logarithmic divergences are then extracted by applying coordinate space techniques.

The crucial observation is that the integrals can be written as products of Green's functions and a summation over lattice sites is performed. By subtracting the leading terms of the asymptotic form of the Green's function one can expand the products of Green's functions in the mass $m$ of the scalar field up to any given order. The coefficients of the logarithmic and quadratic divergences are thus expressed in terms of sums over lattice sites. Precise numerical knowledge of the Green's function values close to the origin and of its asymptotic large- $x$ behavior make an accurate evaluation of these sums possible. Once the external momenta have been eliminated from the propagators (e.g. by applying the BPHZ scheme), the method proposed can be utilized to calculate any two-loop diagram to very high accuracy.

\section{Acknowledgments}

We thank Randy Lewis for useful discussions. We also thank the referee of the first manuscript version for a remark which triggered off the investigation in Sec. 5. Financial support of the Deutsche Forschungsgemeinschaft is gratefully acknowledged.

\section{A Asymptotic expansion of $G(x)$ for large $x$}

In this appendix, we derive the asymptotic expansion of the lattice Green's function $G(x)$ for large $x$. To this aim, it is convenient to introduce the lattice constant $a$ and to study the expansion in $a$ with $\tilde{x}=a x$ fixed which is equivalent to the large $x=\tilde{x} / a$ expansion.

In [6], Paladini and Sexton have studied the asymptotic behavior of the quantity ${ }^{3}$

$$
F_{\alpha}(x)=a^{4-2 \alpha} \int_{-\pi / a}^{\pi / a} \frac{d^{4} k}{(2 \pi)^{4}} e^{i k \cdot \tilde{x}} \frac{1}{\left(\hat{k}^{2}+\mu^{2}\right)^{\alpha}}
$$

where $m=a \mu$, and in this appendix we set

$$
\hat{k}^{n}=\sum_{\nu=1}^{4}\left(\frac{2}{a} \sin \left(\frac{k_{\nu} a}{2}\right)\right)^{n} .
$$

\footnotetext{
${ }^{3}$ In [6] the results were derived in $d$ dimensions, but here we restrict ourselves to $d=4$ dimensions for simplicity.
} 
In the following, we present an alternative and much easier derivation of their results. The small $a$ expansion of the integrand is given by

$$
\begin{aligned}
\frac{1}{\left(\hat{k}^{2}+\mu^{2}\right)^{\alpha}} & =\frac{1}{\left(k^{2}+\mu^{2}\right)^{\alpha}}+a^{2} \frac{\alpha}{12} \frac{k^{4}}{\left(k^{2}+\mu^{2}\right)^{\alpha+1}}+a^{4}\left(\frac{\alpha(\alpha+1)}{288} \frac{\left(k^{4}\right)^{2}}{\left(k^{2}+\mu^{2}\right)^{\alpha+2}}-\frac{\alpha}{360} \frac{k^{6}}{\left(k^{2}+\mu^{2}\right)^{\alpha+1}}\right) \\
& +a^{6}\left(\frac{\alpha(\alpha+1)(\alpha+2)}{10368} \frac{\left(k^{4}\right)^{3}}{\left(k^{2}+\mu^{2}\right)^{\alpha+3}}-\frac{\alpha(\alpha+1)}{4320} \frac{k^{4} k^{6}}{\left(k^{2}+\mu^{2}\right)^{\alpha+2}}+\frac{\alpha}{20160} \frac{k^{8}}{\left(k^{2}+\mu^{2}\right)^{\alpha+1}}\right) \\
& +\mathcal{O}\left(a^{8}\right),
\end{aligned}
$$

such that the original integral reads

$$
F_{\alpha}(x) \simeq F_{\alpha}^{(0)}(x)+a^{2} F_{\alpha}^{(2)}(x)+a^{4} F_{\alpha}^{(4)}(x)+a^{6} F_{\alpha}^{(6)}(x)+\mathcal{O}\left(a^{12-2 \alpha}\right)
$$

with

$$
F_{\alpha}^{(0)}(x)=a^{4-2 \alpha} \int_{-\pi / a}^{\pi / a} \frac{d^{4} k}{(2 \pi)^{4}} e^{i k \cdot \tilde{x}} \frac{1}{\left(k^{2}+\mu^{2}\right)^{\alpha}}
$$

and

$$
\begin{aligned}
& F_{\alpha}^{(2)}(x)=\frac{\alpha}{12} \partial_{\tilde{x}}^{4} F_{\alpha+1}^{(0)}(x) \\
& F_{\alpha}^{(4)}(x)=\frac{\alpha(\alpha+1)}{288}\left(\partial_{\tilde{x}}^{4}\right)^{2} F_{\alpha+2}^{(0)}(x)+\frac{\alpha}{360} \partial_{\tilde{x}}^{6} F_{\alpha+1}^{(0)}(x) \\
& F_{\alpha}^{(6)}(x)=\frac{\alpha(\alpha+1)(\alpha+2)}{10368}\left(\partial_{\tilde{x}}^{4}\right)^{3} F_{\alpha+3}^{(0)}(x)+\frac{\alpha(\alpha+1)}{4320} \partial_{\tilde{x}}^{4} \partial_{\tilde{x}}^{6} F_{\alpha+2}^{(0)}(x)+\frac{\alpha}{20160} \partial_{\tilde{x}}^{8} F_{\alpha+1}^{(0)}(x) .
\end{aligned}
$$

The derivative operators in Eqs. (A.6) are defined by $\partial_{\tilde{x}}^{n}=\sum_{\nu=1}^{4} \partial^{n} / \partial \tilde{x}_{\nu}^{n}$. The crucial observation is that the extension of the integral boundaries in Eq. (A.5) to infinity does not affect the large- $x$ behavior of the integral but merely changes its behavior near the origin. For our purposes we can thus set for $x \rightarrow \infty$

$$
F_{\alpha}^{(0)}(x)=a^{4-2 \alpha} \int_{-\infty}^{\infty} \frac{d^{4} k}{(2 \pi)^{4}} e^{i k \cdot \tilde{x}} \frac{1}{\left(k^{2}+\mu^{2}\right)^{\alpha}}=\left(\frac{a^{2} \mu}{|\tilde{x}|}\right)^{2-\alpha} \frac{1}{2^{\alpha+1} \pi^{2} \Gamma(\alpha)} K_{2-\alpha}(|\tilde{x}| \mu),
$$

where $|\tilde{x}|=\sqrt{\tilde{x}^{2}}$. After evaluating the derivatives in Eq. (A.6) we arrive at the results of [6]. The asymptotic Green's function $G_{a s}(x)$ is immediately obtained from (A.6) by setting $\alpha=1$ :

$$
G_{a s}(x) \simeq G_{a s}^{(0)}(x)+G_{a s}^{(2)}(x)+G_{a s}^{(4)}(x)+G_{a s}^{(6)}(x)+\mathcal{O}\left(a^{10}\right)
$$


with

$$
\begin{aligned}
G_{a s}^{(0)}(x) & =\frac{1}{4 \pi^{2} x^{2}} m|x| K_{1}(m|x|), \\
G_{a s}^{(2)}(x) & =\frac{1}{96 \pi^{2}\left(x^{2}\right)^{2}}\left[12(m|x|)^{2} K_{2}(m|x|)-6(m|x|)^{3} K_{3}(m|x|)+(m|x|)^{4} \frac{x^{4}}{\left(x^{2}\right)^{2}} K_{4}(m|x|)\right] \\
G_{a s}^{(4)}(x) & =\frac{1}{23040 \pi^{2}\left(x^{2}\right)^{3}}\left[2160(m|x|)^{3} K_{3}(m|x|)-2280(m|x|)^{4} K_{4}(m|x|)\right. \\
& +\left(180+840 \frac{x^{4}}{\left(x^{2}\right)^{2}}\right)(m|x|)^{5} K_{5}(m|x|)-\left(60 \frac{x^{4}}{\left(x^{2}\right)^{2}}+72 \frac{x^{6}}{\left(x^{2}\right)^{3}}\right)(m|x|)^{6} K_{6}(m|x|) \\
& \left.+5(m|x|)^{7} \frac{\left(x^{4}\right)^{2}}{\left(x^{2}\right)^{4}} K_{7}(m|x|)\right], \\
G_{a s}^{(6)}(x) & =\frac{1}{11612160 \pi^{2}\left(x^{2}\right)^{4}}\left[1179360(m|x|)^{4} K_{4}(m|x|)-1935360(m|x|)^{5} K_{5}(m|x|)\right. \\
& +\left(241920+1270080 \frac{x^{4}}{\left(x^{2}\right)^{2}}\right)(m|x|)^{6} K_{6}(m|x|)-\left(7560+138600 \frac{x^{4}}{\left(x^{2}\right)^{2}}\right. \\
& \left.+260064 \frac{x^{6}}{\left(x^{2}\right)^{3}}\right)(m|x|)^{7} K_{7}(m|x|)+\left(3780 \frac{x^{4}}{\left(x^{2}\right)^{2}}+16380 \frac{\left(x^{4}\right)^{2}}{\left(x^{2}\right)^{4}}+9072 \frac{x^{6}}{\left(x^{2}\right)^{3}}\right. \\
& \left.+16200 \frac{x^{8}}{\left(x^{2}\right)^{4}}\right)(m|x|)^{8} K_{8}(m|x|)-\left(630 \frac{\left(x^{4}\right)^{2}}{\left(x^{2}\right)^{4}}+1512 \frac{x^{4} x^{6}}{\left(x^{2}\right)^{5}}\right)(m|x|)^{9} K_{9}(m|x|) \\
& \left.+35 \frac{\left(x^{4}\right)^{3}}{\left(x^{2}\right)^{6}}(m|x|)^{10} K_{10}(m|x|)\right) .
\end{aligned}
$$

The leading asymptotic piece in the small $a$ expansion, $G_{a s}^{(0)}$, is (up to a prefactor of $1 / a^{2}$ ) equivalent to the continuum Green's function, while the remaining terms $G_{a s}^{(2 i)}, i \geq 1$, represent corrections of order $a^{2 i}$ with respect to $G_{a s}^{(0)}$ and vanish in the continuum limit $a \rightarrow 0$. 


\section{B Coefficients $\kappa$ and $\lambda$}

In this appendix, we present the coefficients $\kappa$ and $\lambda$ which appear in Eqs. (29), (35) and (62). The coefficients $\kappa$ in Eq. (29) are given in $d$ dimensions by

$$
\begin{aligned}
& \kappa_{0}=\frac{2^{1-3 d} m^{-6+3 d} \pi^{2-\frac{3 d}{2}} \Gamma\left(4-\frac{3 d}{2}\right) \sin \left(\frac{3 d \pi}{2}\right)}{\Gamma\left(2-\frac{d}{2}\right)^{3} \sin \left(\frac{d \pi}{2}\right)^{3}} \\
& \kappa_{1}=\frac{2^{1-3 d} m^{-6+3 d} \pi^{2-\frac{3 d}{2}}\left(-3 \Gamma\left(5-\frac{3 d}{2}\right) \Gamma\left(2-\frac{d}{2}\right)+\Gamma\left(4-\frac{3 d}{2}\right) \Gamma\left(3-\frac{d}{2}\right)\right) \sin \left(\frac{3 d \pi}{2}\right)}{\Gamma\left(2-\frac{d}{2}\right)^{3} \Gamma\left(3-\frac{d}{2}\right) \sin \left(\frac{d \pi}{2}\right)^{3}}, \\
& \kappa_{2}=\frac{2^{-3 d} m^{-6+3 d} \pi^{2-\frac{3 d}{2}}\left(-6 \Gamma\left(5-\frac{3 d}{2}\right) \Gamma\left(2-\frac{d}{2}\right)+\Gamma\left(4-\frac{3 d}{2}\right) \Gamma\left(3-\frac{d}{2}\right)\right) \sin \left(\frac{3 d \pi}{2}\right)}{\Gamma\left(2-\frac{d}{2}\right)^{3} \Gamma\left(3-\frac{d}{2}\right) \sin \left(\frac{d \pi}{2}\right)^{3}} \\
& +\frac{3 \cdot 2^{-3 d} m^{-6+3 d} \pi^{2-\frac{3 d}{2}} \Gamma\left(6-\frac{3 d}{2}\right)\left(\Gamma\left(3-\frac{d}{2}\right)^{2}+2 \Gamma\left(2-\frac{d}{2}\right) \Gamma\left(4-\frac{d}{2}\right)\right) \sin \left(\frac{3 d \pi}{2}\right)}{\Gamma\left(2-\frac{d}{2}\right)^{2} \Gamma\left(3-\frac{d}{2}\right)^{2} \Gamma\left(4-\frac{d}{2}\right) \sin \left(\frac{d \pi}{2}\right)^{3}}, \\
& \kappa_{3}=\frac{2^{-3 d} m^{-6+3 d} \pi^{2-\frac{3 d}{2}}\left((-4+d) \Gamma\left(4-\frac{3 d}{2}\right)+18 \Gamma\left(5-\frac{3 d}{2}\right)\right) \sin \left(\frac{3 d \pi}{2}\right)}{3(-4+d) \Gamma\left(2-\frac{d}{2}\right)^{3} \sin \left(\frac{d \pi}{2}\right)^{3}} \\
& +\frac{2^{4-3 d}(-22+3 d) m^{-6+3 d} \pi^{2-\frac{3 d}{2}} \Gamma\left(7-\frac{3 d}{2}\right) \sin \left(\frac{3 d \pi}{2}\right)}{(-8+d)(-6+d)(-4+d)^{2} \Gamma\left(2-\frac{d}{2}\right)^{3} \sin \left(\frac{d \pi}{2}\right)^{3}}, \\
& \kappa_{4}=-\frac{3 \cdot 2^{5-4 d} m^{-6+3 d} \pi^{\frac{1}{2}-\frac{3 d}{2}} \cot \left(\frac{d \pi}{2}\right) \Gamma\left(\frac{3}{2}-\frac{d}{2}\right)}{-2+d}, \\
& \kappa_{5}=\frac{3 \cdot 2^{2-3 d} m^{-6+3 d} \pi^{1-\frac{3 d}{2}} \cot \left(\frac{d \pi}{2}\right)((-4+d) d \Gamma(3-d)+4 d \Gamma(4-d)+2 \Gamma(5-d))}{(-2+d) d \Gamma\left(3-\frac{d}{2}\right)}, \\
& \kappa_{6}=\frac{3 \cdot 2^{2-3 d} m^{-6+3 d} \pi^{1-\frac{3 d}{2}} \cot \left(\frac{d \pi}{2}\right)((4-d) d(2+d) \Gamma(3-d)+8(5+d) \Gamma(5-d)) \Gamma\left(4-\frac{d}{2}\right)}{(-6+d) d\left(-4+d^{2}\right) \Gamma\left(3-\frac{d}{2}\right)^{2}} \\
& +\frac{3 \cdot 2^{4-3 d}(2+d) m^{-6+3 d} \pi^{1-\frac{3 d}{2}} \cot \left(\frac{d \pi}{2}\right) \Gamma(5-d)}{(-6+d)\left(-4+d^{2}\right) \Gamma\left(3-\frac{d}{2}\right)}, \\
& \kappa_{7}=\frac{3 \cdot 2^{1-3 d} m^{-6+3 d} \pi^{2-\frac{3 d}{2}}}{\Gamma\left(\frac{d}{2}\right)^{2} \sin \left(\frac{d \pi}{2}\right)^{2}}, \\
& \kappa_{8}=\frac{3 \cdot 2^{3-3 d} m^{-6+3 d} \pi^{1-\frac{3 d}{2}} \Gamma\left(3-\frac{d}{2}\right)}{(-2+d) \Gamma\left(1+\frac{d}{2}\right) \sin \left(\frac{d \pi}{2}\right)} \text {. }
\end{aligned}
$$

The coefficients $\lambda$ in Eq. (62) are given by

$$
\begin{aligned}
& \lambda_{0}=0, \\
& \lambda_{1}=-\frac{2^{-1-3 d} m^{-8+3 d} \pi^{2-\frac{3 d}{2}} \Gamma\left(5-\frac{3 d}{2}\right)\left(d \Gamma\left(2-\frac{d}{2}\right)+2 \Gamma\left(3-\frac{d}{2}\right)\right) \sin \left(\frac{3 d \pi}{2}\right)}{\Gamma\left(2-\frac{d}{2}\right)^{3} \Gamma\left(3-\frac{d}{2}\right) \sin \left(\frac{d \pi}{2}\right)^{3}}, \\
& \lambda_{2}=-\frac{2^{-1-3 d} m^{-8+3 d} \pi^{2-\frac{3 d}{2}} \Gamma\left(5-\frac{3 d}{2}\right)\left(d \Gamma\left(2-\frac{d}{2}\right)+2 \Gamma\left(3-\frac{d}{2}\right)\right) \sin \left(\frac{3 d \pi}{2}\right)}{\Gamma\left(2-\frac{d}{2}\right)^{3} \Gamma\left(3-\frac{d}{2}\right) \sin \left(\frac{d \pi}{2}\right)^{3}}
\end{aligned}
$$




$$
\begin{aligned}
& +\frac{2^{-3 d} m^{-8+3 d} \pi^{2-\frac{3 d}{2}} \Gamma\left(6-\frac{3 d}{2}\right)\left(d \Gamma\left(2-\frac{d}{2}\right)+3 \Gamma\left(3-\frac{d}{2}\right)\right) \sin \left(\frac{3 d \pi}{2}\right)}{\Gamma\left(2-\frac{d}{2}\right)^{2} \Gamma\left(3-\frac{d}{2}\right)^{2} \sin \left(\frac{d \pi}{2}\right)^{3}} \\
& +\frac{2^{-1-3 d} d m^{-8+3 d} \pi^{2-\frac{3 d}{2}} \Gamma\left(6-\frac{3 d}{2}\right) \sin \left(\frac{3 d \pi}{2}\right)}{\Gamma\left(2-\frac{d}{2}\right)^{2} \Gamma\left(4-\frac{d}{2}\right) \sin \left(\frac{d \pi}{2}\right)^{3}}, \\
& \lambda_{3}=-\frac{2^{-2-3 d} m^{-8+3 d} \pi^{2-\frac{3 d}{2}}(1+2 \cos (d \pi)) \Gamma\left(5-\frac{3 d}{2}\right)\left(d \Gamma\left(2-\frac{d}{2}\right)+2 \Gamma\left(3-\frac{d}{2}\right)\right)}{\Gamma\left(2-\frac{d}{2}\right)^{3} \Gamma\left(3-\frac{d}{2}\right) \sin \left(\frac{d \pi}{2}\right)^{2}} \\
& +\frac{2^{-3 d} m^{-8+3 d} \pi^{2-\frac{3 d}{2}}(1+2 \cos (d \pi)) \Gamma\left(6-\frac{3 d}{2}\right)\left(d \Gamma\left(2-\frac{d}{2}\right)+3 \Gamma\left(3-\frac{d}{2}\right)\right)}{\Gamma\left(2-\frac{d}{2}\right)^{2} \Gamma\left(3-\frac{d}{2}\right)^{2} \sin \left(\frac{d \pi}{2}\right)^{2}} \\
& -\frac{2^{-1-3 d} m^{-8+3 d} \pi^{2-\frac{3 d}{2}}(1+2 \cos (d \pi)) \Gamma\left(7-\frac{3 d}{2}\right)\left(d \Gamma\left(2-\frac{d}{2}\right)+6 \Gamma\left(3-\frac{d}{2}\right)\right)}{\Gamma\left(2-\frac{d}{2}\right) \Gamma\left(3-\frac{d}{2}\right)^{3} \sin \left(\frac{d \pi}{2}\right)^{2}} \\
& +\frac{2^{-1-3 d} d m^{-8+3 d} \pi^{2-\frac{3 d}{2}}(1+2 \cos (d \pi)) \Gamma\left(6-\frac{3 d}{2}\right)}{\Gamma\left(2-\frac{d}{2}\right)^{2} \Gamma\left(4-\frac{d}{2}\right) \sin \left(\frac{d \pi}{2}\right)^{2}} \\
& -\frac{3 \cdot 2^{-1-3 d} m^{-8+3 d} \pi^{2-\frac{3 d}{2}}(1+2 \cos (d \pi)) \Gamma\left(7-\frac{3 d}{2}\right)\left(d \Gamma\left(2-\frac{d}{2}\right)+\Gamma\left(3-\frac{d}{2}\right)\right)}{\Gamma\left(2-\frac{d}{2}\right)^{2} \Gamma\left(3-\frac{d}{2}\right) \Gamma\left(4-\frac{d}{2}\right) \sin \left(\frac{d \pi}{2}\right)^{2}} \\
& -\frac{2^{-2-3 d} d m^{-8+3 d} \pi^{2-\frac{3 d}{2}}(1+2 \cos (d \pi)) \Gamma\left(7-\frac{3 d}{2}\right)}{\Gamma\left(2-\frac{d}{2}\right)^{2} \Gamma\left(5-\frac{d}{2}\right) \sin \left(\frac{d \pi}{2}\right)^{2}}, \\
& \lambda_{4}=-2^{2-4 d} d m^{-8+3 d} \pi^{\frac{1}{2}-\frac{3 d}{2}} \cot \left(\frac{d \pi}{2}\right) \Gamma\left(\frac{3}{2}-\frac{d}{2}\right), \\
& \lambda_{5}=\frac{2^{-1-3 d}(-4+d) d m^{-8+3 d} \pi^{1-\frac{3 d}{2}} \cot \left(\frac{d \pi}{2}\right) \Gamma(3-d)}{\Gamma\left(3-\frac{d}{2}\right)} \\
& +\frac{8^{-d} m^{-8+3 d} \pi^{1-\frac{3 d}{2}} \cot \left(\frac{d \pi}{2}\right)\left(2 d^{2} \Gamma(4-d)+(6+d) \Gamma(5-d)\right)}{(-2+d) \Gamma\left(3-\frac{d}{2}\right)}, \\
& \lambda_{6}=\frac{2^{-1-3 d}(-6+d) d(2+d) m^{-8+3 d} \pi^{1-\frac{3 d}{2}} \cot \left(\frac{d \pi}{2}\right) \Gamma(5-d) \Gamma\left(3-\frac{d}{2}\right)}{(-2+d) \Gamma\left(4-\frac{d}{2}\right)^{2}} \\
& -\frac{2^{-3-3 d}(-6+d)(-4+d) d m^{-8+3 d} \pi^{1-\frac{3 d}{2}} \cot \left(\frac{d \pi}{2}\right) \Gamma(3-d)}{\Gamma\left(4-\frac{d}{2}\right)} \\
& +\frac{2^{-3 d}(-6+d)(-96+d(-12+d(3+d))) m^{-8+3 d} \pi^{1-\frac{3 d}{2}} \cot \left(\frac{d \pi}{2}\right) \Gamma(5-d)}{(-4+d)(-2+d) d \Gamma\left(4-\frac{d}{2}\right)} \\
& \lambda_{7}=\frac{2^{-3 d} d m^{-8+3 d} \pi^{2-\frac{3 d}{2}}}{\Gamma\left(-1+\frac{d}{2}\right) \Gamma\left(\frac{d}{2}\right) \sin \left(\frac{d \pi}{2}\right)^{2}}, \\
& \lambda_{8}=\frac{2^{-3 d}(-2+(-4+d) d) m^{-8+3 d} \pi^{2-\frac{3 d}{2}}}{\Gamma\left(\frac{d}{2}\right)^{2} \sin \left(\frac{d \pi}{2}\right)^{2}} .
\end{aligned}
$$




\section{References}

[1] M. Lüscher and P. Weisz, Nucl. Phys. B 445 (1995) 429 [arXiv:hep-lat/9502017].

[2] B. Borasoy and H. Krebs, Phys. Rev. D 72 (2005) 056003 [arXiv:hep-lat/0508005].

[3] W. Zimmermann, Commun. Math. Phys. 15, 208 (1969)

[4] T. Reisz, Commun. Math. Phys. 117 (1988) 79.

[5] T. Reisz, Commun. Math. Phys. 116 (1988) 81.

[6] B. Paladini and J. C. Sexton, Phys. Lett. B 448 (1999) 76 [arXiv:hep-lat/9805021].

[7] S. Laporta and E. Remiddi, Nucl. Phys. B 704 (2005) 349 [arXiv:hep-ph/0406160].

[8] S. Pozzorini and E. Remiddi, arXiv:hep-ph/0505041. 